\title{
Correction to: Leading Future Lives: Producing Meaningful Intelligence
}

\section{Correction to:}

Chapter 4 in: P. Bloom, Identity, Institutions and Governance in an $A I$ World, https://doi.org/10.1007/978-3-030-36181-5_4

The original version of the book was inadvertently published with a reference being attributed for the book, My Fair Ladies.

The updated reference is as follows:

Wosk, J. (2015). My Fair Ladies: Female Robots, Androids, and Other Artificial Eves. Rutgers University Press.

The updated version of the chapter can be found at https://doi.org/10.1007/978-3-030-36181-5_4 